\title{
The Effect of Co-dependent (Thinking in Motion [tim]) Versus Single-modality (Cognifit) Interventions on Cognition and Gait Among Community Dwelling Older Adults With Cognitive Decline: a Randomized Controlled Study
}

Shiri Embon-Magal

University of Haifa

Tal Krasovsky

University of Haifa

Israel Doron

University of Haifa

Kfir Asraf

University of Haifa

Iris Haimov

The Max Stern Academic College of Emek Yizreel

Efrat Gil

Technion - Israel Institute of Technology

Maayan Agmon ( $\square$ magmon@univ.haifa.ac.il)

University of Haifa

\section{Research Article}

Keywords: Brain aging, Motor-cognitive intervention, Remedial intervention

Posted Date: January 6th, 2022

DOI: https://doi.org/10.21203/rs.3.rs-707056/v1

License: (9) This work is licensed under a Creative Commons Attribution 4.0 International License.

Read Full License 


\section{Abstract}

Background. Cognition and motor skills are interrelated throughout the aging process and often show simultaneous deterioration among older adults with cognitive decline. Co-dependent training has the potential to ameliorate both domains; however, its effect on the gait and cognition of older adults with cognitive decline has yet to be explored. The aim of this study is to compare the effects of the wellestablished single-modality cognitive computerized training program, CogniFit, with "Thinking in motion (TIM)," a co-dependent group intervention, among community-dwelling older adults with cognitive decline.

Methods. Employing a single-blind randomized control trial design, 47 community-dwelling older adults with cognitive decline were randomly assigned to eight-week thrice weekly trainings of TIM or CogniFit. Pre- and post-intervention assessments included cognitive performance, evaluated by a CogniFit battery, as a primary outcome, and gait, under single- and dual-task conditions, as a secondary outcome.

Results. CogniFit total Z scores significantly improved from baseline to post-intervention for both groups. There was a significant main effect for time $\left[F(1,44)=17.43, p<.001, \eta_{p}{ }^{2}=.283\right]$ but not for group [F ( 1 , $44)=0.001, p=.970]$. No time $X$ group interaction $[F(1,44)=1.29, p=.261]$ was found. No changes in gait performance under single and dual-task performance were observed in both groups.

Conclusions. The findings show that single-modality (CogniFit) and co-dependent (TIM) trainings improve cognition but not gait in older adults with cognitive decline. Such investigations should be extended to include various populations and a broader set of outcome measurements.

Trial registration: anzctr Id: 371522. Date: 08/11/2016

\section{Literature Review}

The older adult population is expected to grow 6-7\% per year between 2025-2029, resulting in a quadrupling in this century. ${ }^{1}$ In 2002, it was estimated that $22 \%$ of adults aged 70 and older in the United States suffer from cognitive impairment without dementia. ${ }^{2}$ Cognitive decline is associated with an increased risk for disability, higher risk for dementia, ${ }^{2}$ reduced gait quality and increased risk of falls. ${ }^{2-4}$ Owing to the interplay between motor and cognitive aging, both cognitive and motor interventions have reciprocal positive effects among older adults. ${ }^{5-7}$ Such interventions can be classified as single-modality (motor or cognitive) or combined training (motor and cognitive) ${ }^{6-7}$ Indeed, single-modality cognitive intervention shows transfer effects to motor abilities ${ }^{5}$ and vice versa. ${ }^{8}$ However, the multi-sensory simultaneous reinforcement of the combined multi-modality training may have a greater effect, especially in low-functioning older adults. ${ }^{9}$

The combined multi-modality motor-cognitive interventions can be divided into three types based on the relationships between tasks: (1) independent performance of serial motor and cognitive tasks (either in 
the same session or in separate sessions), a sequential training shown to be effective mainly when an aerobic motor part precedes the cognitive part of the training ${ }^{10}$; (2) simultaneous performance of tasks i.e., Dual-Task (DT), aiming to improve mainly divided attention ${ }^{3}$, found to be effective in improving various motor (e.g., balance and postural control) and cognitive functions (e.g., executive function, memory, and attention) during single and DT conditions ${ }^{11}$ (however, a systematic review argues that this training has limited transferability ${ }^{10}$ ); and (3) simultaneous performance of co-dependent tasks i.e., an integration of cognitive and motor demands embedded within one task (which cannot be executed separately as in DT), like walking while navigating. ${ }^{12}$

Co-dependent tasks represent the requirements of many daily life tasks and are more ecological than other combined trainings such as DT. ${ }^{12}$ Although understudied compared to the other two types, codependant interventions showed improvement in global cognition ${ }^{13}$, executive control, processing speed $^{14}$, and balance and motor performance ${ }^{15-18}$ among community-dwelling older adults. The potential contribution of co-dependent training to older adults with cognitive decline, though, is yet to be determined. ${ }^{19}$

According to the patient-centered care approach, matching between interventions and specific patients' needs can optimize the intervention's effects. ${ }^{20}$ Moreover, a simultaneous reinforcement of multisensory systems as expressed in the co-dependant training can lead to a greater effect on various systems compared to a single modality training especially among older adults. ${ }^{9}$ To address this question among community-dwelling older adults with cognitive decline, we developed a co-dependant group training with high adaptability to a variety of cognitive levels called "Thinking in Motion - TIM"21 and compared its effect on cognition and gait performance to the well-established cognitive computerized training i.e., CogniFit, among community-dwelling older adults aged 65 and above.

Our primary hypothesis was that the TIM intervention will be as effective as CogniFit in improving global cognition and more specifically in domains such as working memory, divided attention, processing speed, and visual scanning. These domains were intensively trained in TIM intervention and were shown to be improved after Cognifit training. ${ }^{22}$ In addition, considering the specificity of the training of gross motor skills in the TIM intervention, our secondary hypothesis was that TIM will have a higher effect on gait speed and variability during single task (ST) and DT compared to CogniFit.

\section{Methods}

\section{Eligibility and Study Design}

A single-blind, randomized, and controlled clinical trial compared the effects of TIM, a co-dependent motor-cognitive intervention, and CogniFit, a single modality computerized cognitive training (control), on cognition and gait quality in 47 community-dwelling older adults who attend an adult day center (based on $\mathrm{G}$ power calculation on the main outcome cognitive performance). Potential participants were 
recruited through referrals from day center staff members. Inclusion criteria were: (1) age over 65; (2) ability to walk independently with or without an assistive device; (3) ability to understand simple instructions and sign an informed consent form; (4) ability to commit to the program based on a short interview. This set of minimum criteria seeks to represent as reliably as possible the population of the day centers as it is, although indeed, some of the participants demonstrated cognitive decline without a documented dementia diagnosis. Participants who met the inclusion criteria were randomly assigned to an eight-week, thrice per week, 40-minute intervention in one of two groups: TIM group intervention $(n=28)$ or individual computerized cognitive training using CogniFit training ( $n=19)$ (i.e., control group).

Randomization was conducted using a random number table. Baseline assessment was conducted by a research assistant, naive to participant assignment, and included: (1) demographic and psychological measures: The Geriatric Depression Scale (GDS) ${ }^{23}$, Short Anxiety Screening Test (SAST) ${ }^{24}$ and the Activities-specific Balance Confidence (ABC) Scale ${ }^{25}$; (2) cognitive performance: Montreal Cognitive Assessment; $\mathrm{MoCA}^{26}$ and a computerized neuro-cognitive assessment using CogniFit battery ${ }^{27}$ and (3) gait assessment in single and dual-task conditions using an accelerometer and gyroscope mounted on the waist from McRoberts Mobility Lab ${ }^{28}$. Cognitive and gait evaluations were conducted again after eight weeks of intervention. The study protocol was approved by an institutional review board. All participants signed a consent form and did not receive any monetary compensation for participation.

\section{Thinking in Motion (TIM) intervention}

TIM is a combined motor-cognitive co-dependent intervention inspired by the Eshkol-Wachman Movement Notation (EWMN), which uses graphic symbols to describe motion. ${ }^{29}$ Unlike the fixed use of graphic symbols in EWMN, TIM separates from the permanent system of symbols and uses the ephemerality of symbols and frequent changes in them as a central tool for cognitive stimulation. The ephemerality and frequent changes require a renewed learning process, coping with coding permanence and mental flexibility. In addition, the use of symbols creates the cognitive challenge of producing movement from interpreting a graphic sign, i.e; visual scanning, information processing, and spatial perception. The symbols represent various components in movement such as organ movement, landmarks in space, time, and rhythm elements. After establishing the given symbols for the exercise, a cognitive challenge is graded by a constant change of the symbols' order. The TIM trainer uses a "manipulation bank" that is applied to various components of the movement or how they are presented graphically. The chosen manipulations enable the TIM trainer to manage the level of difficulty and the variety of cognitive skills required to perform them. An example of TIM training and manipulation options is shown in Figure 1.

\section{Control group- CogniFit intervention}

CogniFit is a computerized cognitive training which was found to be effective in improving cognition and gait. ${ }^{5-22}$ The program is personally tailored based on a 45 -minute baseline assessment. ${ }^{27}$ Each training 
session includes a mixture of auditory, visual, and cross-modality tasks aimed at training executive functions, attention, and other cognitive processes. Verbal instructions were written on the screen before each task and then demonstrated by the program ${ }^{30}$. Technical support in operating the software and assistance in understanding the tasks were available.

\section{Outcome Measures Assessments}

Measures were undertaken at baseline and after the eight-week intervention. All assessments were conducted by a blinded research assistant.

\section{Primary Outcome: Cognitive Functions-}

The 40-minute CogniFit computerized neurocognitive assessment battery ${ }^{27}$ was used to evaluate changes in cognitive function following both interventions. Apart from the global score, the cognitive indicators examined in this study were working memory, divided attention, processing speed, and visual scanning.

\section{Secondary Outcome: Gait Performance-}

Gait measures included gait speed and stride time variability collected under ST and DT conditions with the McRoberts Mobility Lab, an accelerometer and gyroscope attached to the participants' waists. ${ }^{31}$ Gait tasks took place for periods of one minute along a six-meter route in a quiet room. The cognitive task attached to the walking was subtraction by 3 from a random number between 100 and $250 .{ }^{32}$ The order of the tasks was randomized.

\section{Analysis}

All analyses were performed using SPSS 26 (IBM SPSS Statistics, New York, US). Continuous data are presented as mean and standard deviation or median and interquartile range, while categorical data are presented as frequencies (percentage and number of participants). Between-group differences in demographic data were analysed via Mann-Whitney U tests, Pearsons' chi-square test, or Fisher's Exact tests. CogniFit measures at baseline and post-intervention were examined using between-within repeated measures Analysis of variance (ANOVA) and presented as mean \pm standard error. When an interaction was significant, it was followed by t-test post-hoc analyses. Gait outcomes were speed and stride time variability. Effect size estimator was partial eta-squared $\left(\eta p^{2}\right)$ for the ANOVA test. Effect sizes are reported for significant comparisons only.

\section{Results}




\section{Group Characteristics}

A total of 54 participants were referred to the research team by the center staff. Seven participants withdrew from the study before group assignment: five did not meet the inclusion criteria and two declined to participate (Figure 2(. Thus, the sample was comprised of 30 women and 17 men, with a mean age of 81.16 years $(S D=8.23)$. No differences between groups in age, years of education, gender, smoking, cardio-metabolic diseases, stroke, balance efficacy, anxiety, depression, and cognitive abilities at baseline were found (Table 1). 
Table 1

Comparisons of demographic measures between the groups at baseline:

\begin{tabular}{|c|c|c|c|c|}
\hline Parameter & $\begin{array}{l}\text { Control } \\
(n=19)\end{array}$ & $\begin{array}{l}\text { Intervention } \\
(\mathrm{n}=27)\end{array}$ & $U / t / \chi 2(\mathrm{df})$ & $P$ \\
\hline \multirow[t]{2}{*}{ Age } & Median= 82 & Median= 85 & 148 & .197 \\
\hline & $\mathrm{IQR}=14$ & $\mathrm{IQR}=11$ & & \\
\hline \multirow[t]{2}{*}{ Years of Education } & Median $=10$ & Median= 8.5 & 171.5 & .664 \\
\hline & $\mathrm{IQR}=6.5$ & $\mathrm{IQR}=4$ & & \\
\hline$\%$ of Females & $68.42(n=13)$ & $62.96(n=17)$ & $0.29(1)$ & .758 \\
\hline Smoking (not smoking) & $100(n=19)$ & $100(n=27)$ & - & - \\
\hline Falling in the last year (yes) & $42.10(n=8)$ & $51.85(n=14)$ & $0.42(1)$ & .514 \\
\hline Cardio metabolic disease & $52.63(n=10)$ & $77.77(n=21)$ & $3.20(1)$ & .073 \\
\hline Stroke (yes) & $15.78(n=3)$ & $25.92(n=7)$ & $\wedge$ & .488 \\
\hline Balance Efficacy & Median= 58.7 & Median $=60$ & 188.5 & .855 \\
\hline (ABC- scale 0-100) & IQR= 30.4 & $\mathrm{IQR}=45.6$ & & \\
\hline Anxiety & Median= 31.5 & Median= 29 & 135.5 & .167 \\
\hline (SAST-scale 10-40) & $\mathrm{IQR}=5.75$ & $\mathrm{IQR}=6$ & & \\
\hline Depression & Median $=2$ & Median= 4 & 182 & .397 \\
\hline (GDS -scale 0-30) & $\mathrm{IQR}=3.5$ & $\mathrm{IQR}=5.2$ & & \\
\hline Cognition & Median $=16$ & Median= 16 & 229.5 & .553 \\
\hline (MoCA- scale 0-30) & $\mathrm{IQR}=11$ & $\mathrm{IQR}=8$ & & \\
\hline \multirow[t]{2}{*}{ Gait Speed at Baseline } & Mean=0.59 & Mean $=0.47$ & $1.56(43)$ & .125 \\
\hline & $S D=0.34$ & $S D=0.21$ & & \\
\hline \multicolumn{5}{|c|}{$\begin{array}{l}\text { ^Note: Based on Fisher's Exact Test. Cardio metabolic disease defined as at least one of the following } \\
\text { diagnoses: high blood pressure, heart condition or diabetes. Abbreviations: ABC: Activities-specific } \\
\text { Balance Confidence (higher score denote higher confidence); SAST: Short Anxiety Screening Test } \\
\text { (higher score denote higher level of anxiety); GDS: Geriatric Depression Scale (higher score denote } \\
\text { higher level of anxiety); MoCA: Montreal Cognitive Assessment (higher score denotes higher cognitive } \\
\text { performance). }\end{array}$} \\
\hline
\end{tabular}

\section{Primary Outcomes}

Results are presented in Figure 3. Compared with baseline, both groups improved in cognition as measured by the CogniFit test. Analysis of CogniFit total score (Z-scored) revealed a significant main 
effect for time $\left[\mathrm{F}(1,44)=17.43, \mathrm{p}<.001, \eta_{p}{ }^{2}=.283\right]$ such that the cognitive performance at postintervention ( $M=-3.31, S D=1.40)$ improved significantly in both groups, compared to the cognitive performance at baseline $(M=-3.89, S D=1.49)$, with a large effect size. No main effect for group $[F(1,44)$ $=0.001, p=.970]$, or a time $X$ group interaction $[F(1,44)=1.29, p=.261]$ was found. Similar results were demonstrated in specific cognitive domains: working memory following intervention $(M=-3.4, S D=1.36)$ improved compared to baseline $(M=-4.00, S D=1.63)$, with a large effect size $[F(1,44)=10.97, p=.001$, $\left.\eta_{p}^{2}=.199\right]$ with no main effect for group or a time $X$ group interaction. Divided attention improved following intervention with a medium effect size $\left[F(1,44)=5.54, p=.023, \eta_{p}{ }^{2}=.111\right]$ from $(M=-2.28, S D=$ $0.50)$ to $(M=-2.05, S D=0.61)$. No main effect for group was found, but the time $X$ group interaction $[F(1$, $44)=3.90, p=.054]$ was closer to being statistically significant. Processing speed post-intervention $(M=-3.87, S D=2.24)$ improved compared with baseline $(M=-4.50, S D=2.38)$ with a medium effect size $[F$ $\left.(1,41)=5.73, p=.021, \eta_{p}^{2}=.122\right]$, and no effect for group or time $\mathrm{X}$ group interaction. In contrast, in visual scanning domain a significant time $X$ group interaction emerged $\left[F(1,44)=4.63, p=.036, \eta_{p}^{2}\right.$ $=.095]$ with a medium effect size. Post-hoc analysis revealed that while there was no difference between the groups at baseline $\mathrm{t}(44)=-0.13, \mathrm{p}=.893]$, there was a significant difference at post-intervention $[\mathrm{t}(44)=$ $-2.11, p=.039]$, so the TIM group $(M=-1.63, S D=2.03)$ improved more than CogniFit $(M=-2.96, S D=2.17)$. Paired-samples t-tests showed no difference within the groups (not shown).

\section{Secondary outcomes}

Analyses of gait speed under ST condition revealed no main effect for time $[F(1,42)=0.02, p=.876]$ or group $[F(1,42)=1.60, p=.212]$, and no significant time $X$ group interaction $[F(1,42)=2.23, p=.142]$. On the other hand, under DT condition a main effect for group was found $\left[F(1,43)=5.36, p=.025, \eta_{p}{ }^{2}=\right.$ 0.11 , so the control group had a higher mean gait speed $(M=0.41, S D=0.15)$ compared to the intervention group $(M=0.31$ meter / seconds, $S D=0.15)$ at both time points. No main effect for time $[F(1,43)=0.05$, $p=.816]$ or time $X$ group interaction $[F(1,43)=0.02, p=.869]$ were found. Variability of stride time did not differ between or within the groups, regardless of time or condition (Data not shown).

\section{Discussion}

The findings of this single-blind randomized clinical trial demonstrate cognitive improvement in both interventions i.e., TIM and CogniFit among community-dwelling older adults with cognitive decline. Most of our findings support the first hypothesis, suggesting similar global cognitive improvement, as well as similar improvement in specific domains i.e., working memory, divided attention, and processing speed among both groups. These findings are in line with previous findings. ${ }^{13-14-17-22-33}$ One exception is the visual scanning domain, which, in contrast to our hypothesis, was improved only among TIM participants. Indeed, visual scanning is a central requirement within TIM training due to its extensive use in graphical tools. Unlike our findings, a previous study shows improvement in visual scanning following eight weeks of CogniFit training among community-dwelling older adults. ${ }^{22}$ This discrepancy can be explained by the different cognitive abilities of the participants in the current study and Shatils' reseach, ${ }^{22}$ 
probably thus limiting the participants in the control group (Cognifit) from benefiting from its advantages. Notwithstanding, such cognitive results are encouraging especially given the participants' relatively low baseline cognitive scores ${ }^{34}$ and further support the potential for cognitive improvement even among older adults with cognitive impairments. ${ }^{35}$ Such a potential is supported by the remedial model that stresses the ability of training to reinforce brain plasticity. ${ }^{36-37}$

Our second hypothesis, that both groups will show motor improvement with an advantage for the TIM group, was not supported and no significant improvement in gait performance was observed among participants in either group. These findings are inconsistent with a previous study that demonstrates gait improvement after thrice weekly, eight-week CogniFit training among high functioning older adults ${ }^{5}$ and among studies that evaluated the effect of various co-dependent trainings in various older adults populations. ${ }^{15-38}$ The difference between findings may stem from baseline cognitive statues of the participants in the different above mentioned studies. Indeed, the learning abilities of those with cognitive decline are limited. ${ }^{35}$ Although, in the current study, due to safety reasons, the intervention was conducted while sitting, similar to the control group, both groups maintained their gait abilities during the intervention period. Indeed, maintenance of abilities is often the main goal with older adults with cognitive decline. ${ }^{19}$

This study contained several limitations. The first is the sample homogeneity that limits our ability to generalize the findings to different populations, such as higher functioning older adults or patients with neurological diseases. Second, the cognitive assessment was conducted using the Cognifit assessment tool which was similar to the training of CogniFit. Yet, the fact that both groups similarly improved in this test emphasizes the transferability of TIM training. Third, the motor effects of the interventions were examined only on gait in a laboratory setting, limiting its transferability to other daily functions. Fourth, it is hard to infer a causal pathway between the interventions and the preserved gait performance, due to the lack of a waiting list control group. Fifth, the effect of the difference between settings (individual vs group) could not be controlled. Future studies should be conducted among various populations that explore broader motor and social abilities (e.g., participation) in ecological settings and incorporate a waiting list control arm.

\section{Conclusions}

Our findings demonstrate that among community-dwelling older adults with cognitive decline, eight weeks of thrice-weekly interventions (TIM and CogniFit) contribute to global cognition, working memory, divided attention, and processing speed. TIM also demonstrated an improvement in visual scanning. No change in gait performance was observed among participants in both groups. Older adults can benefit from the advantages of both interventions, supporting personalization of treatment plans. Future studies should evaluate TIM among a broader population of older adults in different settings to further address the effect of co-dependent combined training compared to a single modality intervention on motor abilities and cognition throughout the aging process. 


\section{Abbreviations}

Thinking In Motion- TIM,

dual Task-DT.

\section{Declarations}

\section{Ethics approval and consent to participate:}

The study was approved by the Institutional Review Board of the Faculty of Social Welfare and Health Sciences, University of Haifa. All methods were performed in accordance with regulations. All participants sign an inform consent and did not receive any monetary compensation for participation. Study subjects reported general satisfaction with participation in the study, no injuries or harm were reported.

\section{Consent for publication:}

Not applicable.

\section{Availability of data and material:}

The datasets used and/or analyzed during the current study available from the corresponding author on reasonable request. The study is registered in ANZCTR, under registration number 371522, where the full protocol is available.

\section{Competing interests:}

TIM is a training method that the author Shiri Embon Magal has developed and practiced. Other authors had no competing interests.

Funding: This work was partially supported by the Israeli Alzheimer's Association of Israel and Teva Pharmaceuticals. The support was expressed in the funding of the working hours of the research assistant and the intervention trainer. Amda and Teva did not take any part or role in the study.

\section{Authors' contributions:}

Regarding the authors' contribution:

SEM - Developed the intervention, designed the study, analyzed the data, and wrote the manuscript.

MA- Supervised the study, wrote the manuscript. 
ID- Supervised the study and review the manuscript.

TK-Support mobility data analysis and review the manuscript.

EG-Support cognitive data analysis and review the manuscript.

KA- Support statistical analysis and review the manuscript.

$\mathrm{IH}$-Support Cognifit intervention and review the manuscript.

\section{References}

1. Brunborg H. Increasing life expectancy and the growing elderly population. Norsk Epidemiologi. 2012;22(2)doi:https://doi.org/10.5324/nje.v22i2.1552

2. Plassman BL, Langa KM, Fisher GG, et al. Prevalence of cognitive impairment without dementia in the United States. Annals of internal medicine. 2008;148(6):427-434.

3. Clark DJ, Rose DK, Ring SA, Porges EC. Utilization of central nervous system resources for preparation and performance of complex walking tasks in older adults. Frontiers in aging neuroscience. 2014;6:217.

4. Kennedy KM, Raz N. Age, sex and regional brain volumes predict perceptual-motor skill acquisition. Cortex. 2005;41(4):560-569.

5. Verghese J, Mahoney J, Ambrose AF, Wang C, Holtzer R. Effect of cognitive remediation on gait in sedentary seniors. Journals of Gerontology Series A: Biomedical Sciences and Medical Sciences. 2010;65(12):1338-1343.

6. Bamidis $P$, Vivas A, Styliadis $C$, et al. A review of physical and cognitive interventions in aging. Neuroscience \& Biobehavioral Reviews. 2014;44:206-220.

7. Zhu X, Yin S, Lang M, He R, Li J. The more the better? A meta-analysis on effects of combined cognitive and physical intervention on cognition in healthy older adults. Ageing Research Reviews. 2016;31:67-79.

8. Etgen T, Sander D, Huntgeburth U, Poppert H, Förstl H, Bickel H. Physical activity and incident cognitive impairment in elderly persons: the INVADE study. Archives of internal medicine. 2010;170(2):186-193.

9. Paraskevoudi N, Balcı F, Vatakis A. "Walking" through the sensory, cognitive, and temporal degradations of healthy aging. Annals of the New York Academy of Sciences. 2018;1426(1):72-92.

10. Law LL, Barnett F, Yau MK, Gray MA. Effects of combined cognitive and exercise interventions on cognition in older adults with and without cognitive impairment: a systematic review. Ageing research reviews. 2014;15:61-75.

11. Agmon M, Belza B, Nguyen HQ, Logsdon RG, Kelly VE. A systematic review of interventions conducted in clinical or community settings to improve dual-task postural control in older adults. Clinical interventions in aging. 2014;9:477. 
12. Herold F, Hamacher D, Schega L, Müller NG. Thinking while moving or moving while thinkingconcepts of motor-cognitive training for cognitive performance enhancement. Frontiers in aging neuroscience. 2018;10:228.

13. Rosenberg D, Depp CA, Vahia IV, et al. Exergames for subsyndromal depression in older adults: a pilot study of a novel intervention. The American Journal of Geriatric Psychiatry. 2010;18(3):221-226.

14. Maillot P, Perrot A, Hartley A. Effects of interactive physical-activity video-game training on physical and cognitive function in older adults. Psychology and aging. 2012;27(3):589.

15. Wong AM, Lin Y-C, Chou S-W, Tang F-T, Wong P-Y. Coordination exercise and postural stability in elderly people: effect of Tai Chi Chuan. Archives of physical medicine and rehabilitation. 2001;82(5):608-612.

16. Kimura K, Hozumi N. Investigating the acute effect of an aerobic dance exercise program on neurocognitive function in the elderly. Psychology of Sport and Exercise. 2012;13(5):623-629.

17. Pohl P, Dizdar N, Hallert E. The Ronnie Gardiner Rhythm and Music Method-a feasibility study in Parkinson's disease. Disability and rehabilitation. 2013;35(26):2197-2204.

18. Mirelman A, Rochester $L$, Maidan I, et al. Addition of a non-immersive virtual reality component to treadmill training to reduce fall risk in older adults (V-TIME): a randomised controlled trial. The Lancet. 2016;388(10050):1170-1182.

19. Smith-Ray RL, Hughes SL, Prohaska TR, Little DM, Jurivich DA, Hedeker D. Impact of cognitive training on balance and gait in older adults. Journals of Gerontology Series B: Psychological Sciences and Social Sciences. 2015;70(3):357-366.

20. Association AP, Organizations AoP. Psychodynamic diagnostic manual (PDM). Interdisciplinary Council on; 2006.

21. Motion Ti. Thinking in Motion. Accessed July 9, 2021, 2021. https://www.tim-method.com/thinkingin-motion)

22. Shatil E. Does combined cognitive training and physical activity training enhance cognitive abilities more than either alone? A four-condition randomized controlled trial among healthy older adults. Frontiers in aging neuroscience. 2013;5:8.

23. Nyunt MSZ, Fones C, Niti M, Ng T-P. Criterion-based validity and reliability of the Geriatric Depression Screening Scale (GDS-15) in a large validation sample of community-living Asian older adults. Aging and Mental Health. 2009;13(3):376-382.

24. Sinoff G, Ore L, Zlotogorsky D, Tamir A. Short anxiety screening test-a brief instrument for detecting anxiety in the elderly. International journal of geriatric psychiatry. 1999;14(12):1062-1071.

25. Powell LE, Myers AM. The activities-specific balance confidence (ABC) scale. The Journals of Gerontology Series A: Biological Sciences and Medical Sciences. 1995;50(1):M28-M34.

26. Nasreddine Z, Phillips N, Bedirian V. The Montreal Cognitive Assessment, MoCA: a brief screening tool for mild cognitive impairment. JAm Geriatr Soc 2005; 53: 695-9. Mioshi E, Dawson K, Mitchell J, Arnold R, Hodges J. The Addenbrooke's Cognitive Examination Revised (ACE-R): a brief cognitive test battery for dementia screening. Int J Geriatr Psychiatry. 2006;21:1078-5.

Page 12/16 
27. Haimov I, Hanuka E, Horowitz Y. Chronic insomnia and cognitive functioning among older adults. Behavioral sleep medicine. 2008;6(1):32-54.

28. Lab MM. McRoberts-Moving Technology Accessed July 8, 2021, 2021. https://www.mcroberts.nl/

29. Eshkol N, Wachman A. Movement Notation. London: Weidenfeld and Fedigan, LM (1982). Primate paradigms Sex roles and social bonds. 1958;

30. CogniFit. Cognitive Research Platform. Accessed July 8, 2021, 2021. https://www.cognifit.com/cognitive-research-tool

31. Senden R, Grimm B, Heyligers I, Savelberg H, Meijer K. Acceleration-based gait test for healthy subjects: reliability and reference data. Gait \& posture. 2009;30(2):192-196.

32. Agmon M, Kelly VE, Logsdon RG, Nguyen H, Belza B. The effects of EnhanceFitness (EF) training on dual-task walking in older adults. Journal of applied gerontology. 2015;34(3):NP128-NP142.

33. Eggenberger $\mathrm{P}$, Schumacher $\mathrm{V}$, Angst $\mathrm{M}$, Theill N, de Bruin ED. Does multicomponent physical exercise with simultaneous cognitive training boost cognitive performance in older adults? A 6-month randomized controlled trial with a 1-year follow-up. Clinical interventions in aging. 2015;10:1335.

34. Narazaki K, Nofuji Y, Honda T, Matsuo E, Yonemoto K, Kumagai S. Normative data for the montreal cognitive assessment in a Japanese community-dwelling older population. Neuroepidemiology. 2013;40(1):23-29.

35. Hill NT, Mowszowski L, Naismith SL, Chadwick VL, Valenzuela M, Lampit A. Computerized cognitive training in older adults with mild cognitive impairment or dementia: a systematic review and metaanalysis. American Journal of Psychiatry. 2017;174(4):329-340.

36. Ben-Yishay Y, Prigatano GP. Cognitive remediation. 1990;

37. Sharma I, Srivastava J, Kumar A, Sharma R. Cognitive remediation therapy for older adults. Journal of Geriatric Mental Health. 2016;3(1):57.

38. Nitz J, Kuys S, Isles R, Fu S. Is the Wii Fit ${ }^{\text {TM }}$ a new-generation tool for improving balance, health and well-being? A pilot study. Climacteric. 2010;13(5):487-491.

\section{Figures}


Figure 1 - Example of manipulation options in TIM exercise

Base Exercise:

The practitioner is presented with the following table (table 1). In this example the table consists of two rows, each representing a different organ participating in the motor task. The table is read from left to right; the reading is of the whole column at once.

The signs used here are can arrows pointing up and down indicating the direction of hand movement. Other signs can be used, for example non-associative signs like $\mathrm{X}=\mathrm{UP}$ $\mathrm{O}=\mathrm{DOWN}$ or use of arrows but with inverse meaning (Arrow facing up meaning - hand descending and arrow facing down - hand rising).

In table 1, the up arrows marked in the first column in both rows indicates that both hands are raised simultaneously. In the next column one arrow in the right-hand column indicates that just the right hand is moving down, etc.

Table 1

\begin{tabular}{|l|l|l|l|l|l|l|l|}
\hline right hand & $\uparrow$ & $\downarrow$ & & $\uparrow$ & & $\downarrow$ & $\uparrow$ \\
\hline left hand & $\uparrow$ & & $\downarrow$ & & $\uparrow$ & $\downarrow$ & $\uparrow$ \\
\hline
\end{tabular}

Example for manipulation on moving organs:

Addition of leg movement. ( legs move from the knee down, exercise is performed in a sitting position).

Signs instructions: Solo arrow- just hands are moving.

Arrow surrounded by a circle- Matching leg moves with hand

Arrow surrounded by a square- Opposite leg moves with hand (table 5)

Table 2

\begin{tabular}{|l|c|c|c|c|c|c|c|}
\hline right hand & $\uparrow$ & $\downarrow$ & $\uparrow$ & $\downarrow$ & & $\uparrow$ & $\downarrow$ \\
\hline left hand & & $\uparrow$ & & $\downarrow$ & $\uparrow$ & $\emptyset$ & \\
\hline
\end{tabular}

\section{Figure 1}

Please See image above for figure legend. 
Figure 2: CONSORT Flow of participant's diagram

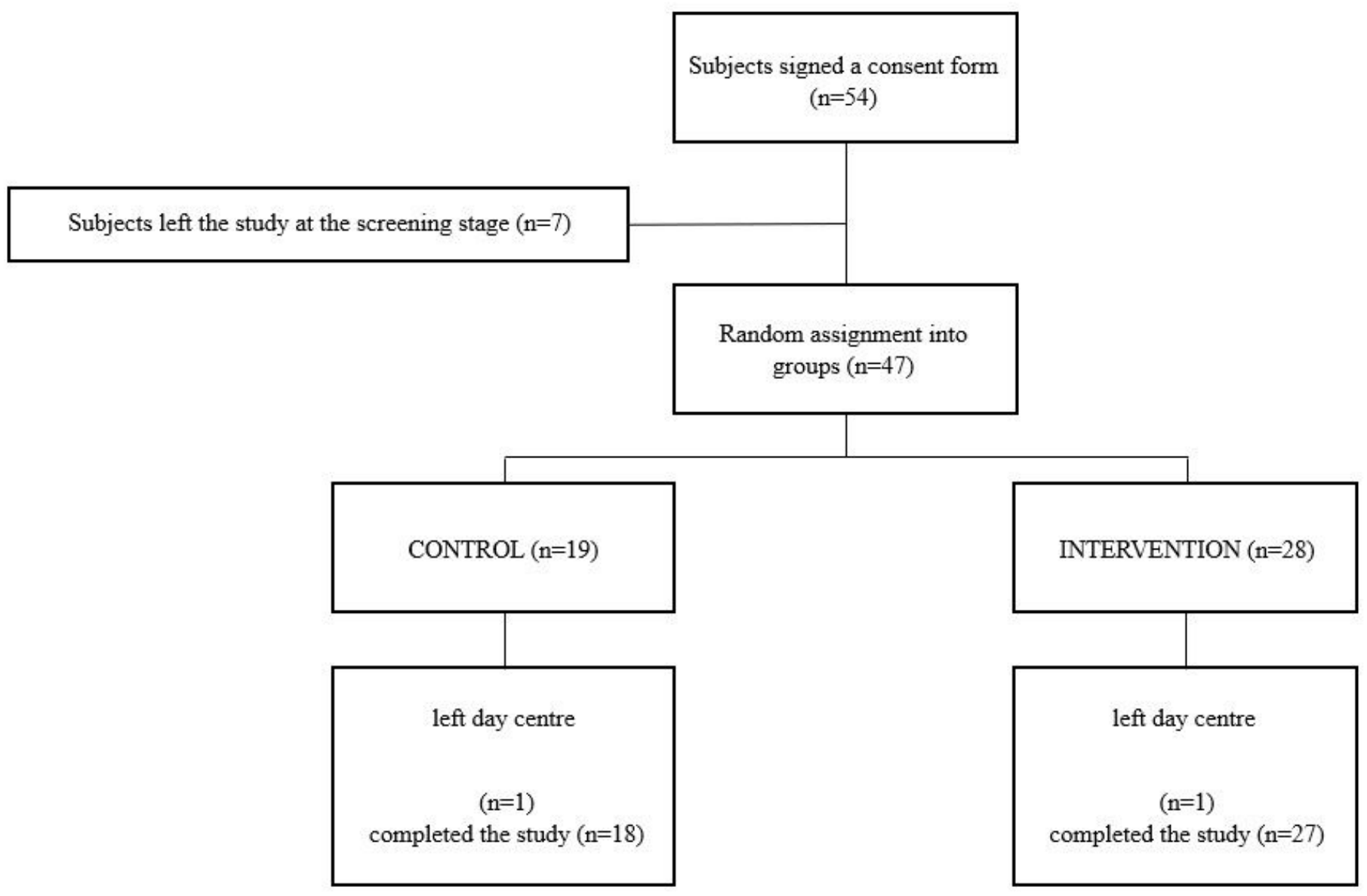

Figure 2

Please See image above for figure legend. 
Figure 3: Effect of interventions on global cognition and on specific domains

A. Global cognition

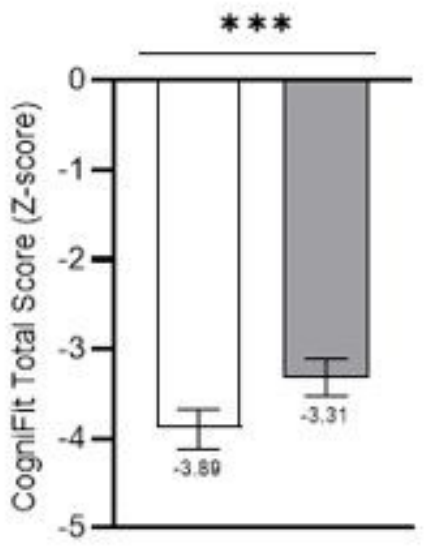

C. Divided Attention

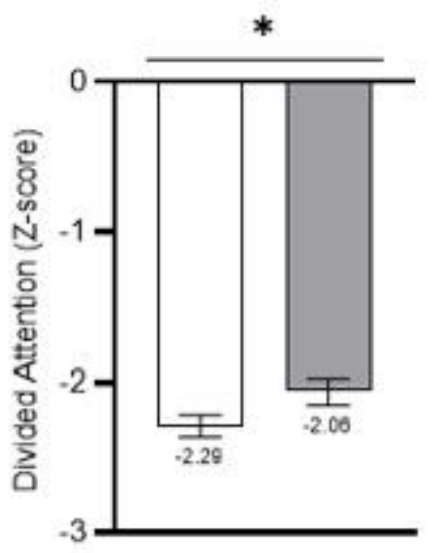

B. Working Memory

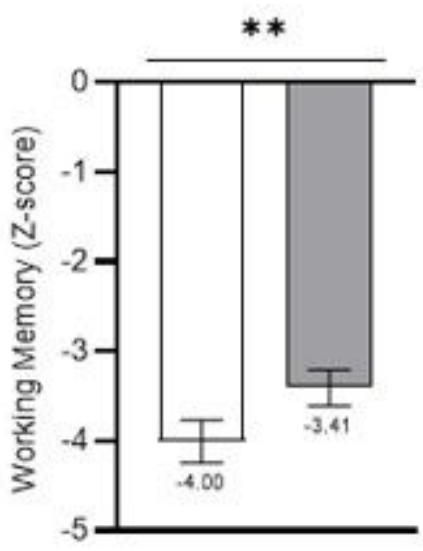

D. Processing Speed

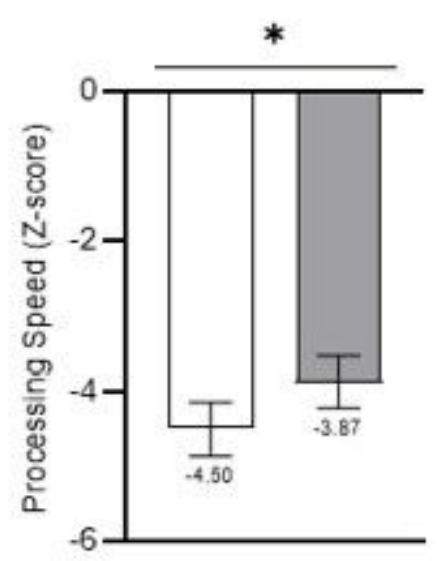

Baseline

Post-Intervention

Figure $3:{ }^{*} p<.05,{ }^{* *} p<.01, * * * p<.001$. Lower numbers indicate worse cognitive performance.

Figure 3

Please See image above for figure legend. 